\title{
CRISPR/Cas9 基因编辑技术在脑科学中的应用策略
}

\author{
张骑鹏 ${ }^{1,2}$, 孙安阳 ${ }^{1^{*}}$ \\ 1. 上海健康医学院脑退行性疾病重点实验室, 上海 201318; \\ 2. 南京大学生命科学学院, 南京 210023 \\ *联系人, E-mail: sunay@sumhs.edu.cn
}

2017-06-15 收稿, 2017-08-14 修回, 2017-08-17 接受, 2017-09-14 网络版发表

摘要 基因编辑是对生物体基因组的目标基因进行精确切割、插入等操作. CRISPR/Cas9技术是基于向导RNA 识别DNA靶序列, Cas9蛋白作为核酸酶切割DNA靶点来实现基因编辑. 该技术自2012年报道以来已被不断改进, 因具有普适、高效、简便等优点, 迅速成为现阶段应用最广的基因编辑技术. 在脑科学领域, CRISPR/Cas9技术不 仅可应用于离体神经细胞，也可以在受精卵期、胚胎期或成年期应用; 应用目的涉及脑基因与功能研究、基因敲 除/敲入小鼠模型的构建、某些疾病的实验性治疗等. 尤其是在一些遗传性疾病如视网膜色素变性、亨廷顿病的动 物模型上, CRISPR/Cas9方法已经初步展示了令人鼓舞的治疗效果. 未来, 该技术将会在精确编辑效率与可控性方 面有进一步提升，并可能在脑定向导入方法、脑神经环路解析等专业应用环节获得显著的发展.

关键词 CRISPR/Cas9, 基因编辑, 基因敲除, 基因敲入, 神经科学

20世纪80年代，第一只转基因小鼠的诞生标志 着人类在哺乳动物上进行基因修饰的开始. 初代转 基因技术是采用原核注射或病毒感染等手段将外源 性DNA导人细胞核，再经随机插人的方式整合进受 体生物的基因组. 随后又通过同源重组机制 ${ }^{[1]}$ 发展了 基因打靶技术, 以提高基因修饰的精度. 迄今, 经由 胚胎干细胞基因打靶方法已成功制备了上千种基因 突变小鼠, 极大地推动现代生物学与医学的发展. 然 而, 基因打靶技术操作费时费力, 制约了它成为一种 普适、快捷的基因修饰技术常规应用.

基于识别DNA靶点的方法不同, 近十余年主要 产生了3种位点特异性基因编辑技术：锌指蛋白核酸 酶(zinc-finger nuclease, ZFN)、转录激活样效应因子 核酸酶(transcription activator-like effector nuclease, TALEN)和成簇的规律间隔短回文重复序列/相关蛋 白9 (CRISPR/Cas9)技术. ZFN和TALEN都是由经设 计改造的DNA识别蛋白与FokI非特异性核酸酶结构
域连接而成, 后者要以二聚体的形式发挥酶切效应. ZFN的每个锌指蛋白识别并结合DNA链上一个特异 的三联碱基，因是一对三的方式，在设计多个锌指蛋 白串联排列时需要考虑上下文关联效应. TALEN技 术的每个TALE蛋白结构单元可识别DNA链上一个 碱基，因是一对一的方式，所以设计更加灵活，可靶 向较长序列. 然而, 大量高度保守的TALE蛋白模块 组装涉及繁琐的分子克隆技术，模块序列高度重复 也可能妨碍后期的病毒包装. CRISPR/Cas9技术采用 向导RNA识别DNA靶序列，以 Cas9蛋白作为核酸酶 切割DNA靶点. 很显然, 这种依靠RNA识别DNA靶 序列的技术比前两种以蛋白识别DNA靶序列的技术 更为简便，加上一些其他优点 (表 1 ), 使之迅速成为 广泛应用的基因编辑技术 ${ }^{[2]}$. 新近，国际上又有基因 编辑技术改进的报道, 如CRISPR/CasX和CRISPR/ $\mathrm{Cas} \mathrm{Y}^{[3]}, \mathrm{CRISPR} / \mathrm{Cpf} 1^{[4]}$, 结构识别的核酸酶 $\mathrm{SGN}^{[5]}$ 等, 但这些改进型技术的编辑效率和可靠性尚待以 


\section{表 1 常见基因编辑技术的特点比较}

Table 1 Comparison of three major techniques for genome editing

\begin{tabular}{lccc}
\hline & ZFN & TALEN & CRISPR/Cas9 \\
\hline 编辑效率 & 低 & 中 & 高 \\
序列特异性 & $\begin{array}{c}\text { 低, 有上下文 } \\
\text { 关联效应 }\end{array}$ & 高 & 高 \\
多基因编辑 & 困难, 涉及全 & 困难, 涉及全 & 简便, 仅需一次应 \\
& 套设计 & 套设计 & 用几个gRNA \\
成本 & 高 & 高 & 低 \\
\hline
\end{tabular}

后更多的实验室去使用与验证. 本文就CRISPR/Cas9 技术在神经科学中的应用及发展, 进行总结与分析.

\section{CRISPR/Cas9系统的发现}

1987年，日本学者在克隆大肠杆菌碱性磷酸酶 同工酶 $i a p$ 基因时, 意外发现该基因下游存在串联的 $29 \mathrm{nt}$ 重复序列, 被 $32 \mathrm{nt}$ 不重复序列间隔开来 ${ }^{[6]}$, 但一 直不清楚这种结构的生物学意义. 此后, 在不同的细 菌和古细菌中发现了更多的这类成簇的规律间隔短 回文重复(clustered regularly interspaced short palindromic repeats, CRISPR)序列, 这类序列存在于大约 $40 \%$ 的细菌和 $90 \%$ 的古细菌中. 另外, 在 CRISPR序 列的上游又发现了与之相关联的Cas (CRISPR-associated) 基因家族, 编码核酸酶和解旋酶等产物与 CRISPR共同发挥作用. 细菌和古细菌中的CRISPR/ Cas系统可分为 3 种类型, 目前应用最广泛的系统属 于 II 型, 包含特征性的Cas9蛋白. II 型系统仅存在于 细菌中, 需要 Cas9蛋白与 crRNA (CRISPR-derived RNA)和tracrRNA (trans-activating RNA)两种RNA共 同参与, 以完成对噬菌体病毒DNA的切割. 随后的 研究表明, crRNA和tracrRNA可以融合在一条RNA单 链上, 由这条RNA单链引导 Cas9蛋白识别DNA靶点, 因此称之为向导RNA (guide RNA, gRNA) ${ }^{[7]}$. 自 2013 年以来, II 型Cas9系统被不断证明具有广泛的物种 有效性, 从最初的宿主嗜热链球菌扩展到了其他种 属, 并包括哺乳类细胞, 从而使CRISPR/Cas9技术的 应用领域也迅速扩大 ${ }^{[8]}$.

\section{CRISPR/Cas9系统的基因编辑机制}

\section{1 对靶基因的位点识别要求}

基础的CRISPR/Cas9系统由长度约100个核苷酸 (5'端20个核苷酸结合靶序列)的 gRNA与Cas9核酸酶
组成, Cas9核酸酶切割靶基因的特异性由gRNA序列 决定. DNA靶序列的下游方向 (对应于gRNA的3'端) 还需有紧邻着的PAM (protospacer-adjacent motif)基 序, 可被Cas9酶识别. 对于广泛使用的化脓链球菌 Cas9酶(SpCas9), PAM基序是NGG (N代表任意一种 碱基). 需要指出的是, 不同种属的Cas9酶识别不同 的PAM基序。例如，金黄色葡萄球菌的SaCas9酶识 别的PAM基序为 NGRRT或NGRRN ( $R$ 代表 $A$ 或 $G$ 碱 基). 通过定向进化方法, 寻找到几个Cas9变种, 可 以识别新的PAM基序, 进而扩大了可识别靶序列的 范围 ${ }^{[9]}$.

\subsection{Cas9核酸酶切割DNA的机制}

Cas9核酸酶由识别叶REC和核酸酶叶NUC组成, 其中核酸酶叶又包含 $\mathrm{HNH}$ 和RuvC两个结构域, 酶活 性状态由构象控制 ${ }^{[10,11]}$. gRNA的骨架区与Cas9表面 带正电荷的沟槽相互作用形成一个核糖核蛋白 (ribonucleoprotein, RNP)复合物, Cas9转变为DNA结 合构象, 可与基因组上的PAM基序结合. 在靶点识 别过程中, gRNA靶序列的3'端“种子区”首先与一小 段DNA靶序列配对结合, RNA链侵人促使DNA解链; 随着正确配对持续, gRNA沿3'端向 $5^{\prime}$ 端方向“拉链式” 打开DNA靶序列的双链. 此配对与解链过程也触发 Cas9的HNH结构域发生变构激活, 连接部位结构重 排又促成RuvC酶活性. 两个核酸酶结构域在激活过 程中各自靠向切割位点, HNH切割DNA双链中的 gRNA互补链, RuvC则切割非互补链, 确保了协调切 割DNA双链. 部分互补的非靶序列可以结合 Cas9, 但不足以驱动 $\mathrm{HNH}$ 变构激活, 不产生DNA双链切割. 另一方面, $\mathrm{HNH}$ 或RuvC结构域的关键氨基酸残基突 变可使其内切酶活性丧失, 例如SpCas9的D10A突变 导致RuvC失活, H840A突变导致 HNH失活. 仅包含 一个活性结构域的Cas9不产生DNA双链断裂, 只切 割DNA单链, 形成一个切口, 又称为 Cas9切口酶 (Cas9 nickase, nCas9), 如常用的切口酶Cas $9^{\text {D10A 突变 }}$ 体仅HNH结构域有活性. 使用Cas9双切口酶, 配合 一对预设计的gRNAs, 可产生DNA双链断裂.

\subsection{DNA双链断裂的后续效应}

CRISPR/Cas9系统的直接作用是在基因靶序列 中位于PAM基序上游 3 4 nt处产生DNA双链断裂. 20 世纪90年代即发现, 细胞DNA双链断裂可以通过两 
种方式进行修复：一种是非同源性末端接合 (nonhomologous end joining, NHEJ)修复, 另一种是同源 重组修复(homology directed repair, HDR). NHEJ修复 很活跃, 是体内主要的修复方式; 但NHEJ修复易产 生小片段插人、缺失、倒位等多种突变, 可导致可读 框移码或突变成终止密码子, 造成该基因不表达或 表达异常. HDR修复活性通常很低, 但为精确修复, 在 gRNA/Cas9体系中添加修复模板可将模板DNA精 确地插人到靶基因的特定位点. 修复模板包含所需 插人的基因片段以及断裂点上下游同源序列(也称为 同源臂). HDR主要发生在分裂细胞的 $\mathrm{G} 2$ 期和 $\mathrm{S}$ 期, 在 非分裂细胞中HDR几无活性. 一些实验室尝试人为地 调节NHEJ与HDR的相对活性 ${ }^{[12]}$ 或者结合细胞周期同 步化处理, 以提高CRISPR/Cas9技术预期修复方式的 编辑效率，希望可应用于改进基因敲人小鼠的生产.

\section{CRISPR/Cas9技术应用方式}

\section{1 受精卵期应用}

采用CRISPR/Cas9技术构建基因工程模式生物
在理论上比较简便，仅需向特定细胞导人 Cas 9 , gRNA和供体DNA, 就可以引导Cas9介导的DNA编 辑事件. 初始的常用方法是向单细胞期受精卵注射 Cas9 mRNA和gRNA混合物. 但由于Cas9蛋白表达有 一定的时间延后，基因编辑实际上多发生于卵裂后 胚胎发育的早期, 因而只有一部分细胞被编辑, 形成 嵌合体(图1)。这种情况在卵裂较早的生物, 如斑马 鱼等尤易发生. 为了有利于产生非嵌合体型胚胎, 目 前应用于受精卵期的CRISPR/Cas9技术已做了一些 改变: ( i ) 直接将预装配的Cas9活性蛋白与gRNA复 合物导人受精卵, 由于Cas9-gRNA立即起效, 加之 Cas9蛋白的细胞半衰期约为 $24 \mathrm{~h}$, 可以最大程度地减 少嵌合体的形成等后遗效应. 各组分的浓度可通过 预实验优化. (ii) 许多研究给Cas9蛋白加上核定位 信号, 用以提高基因编辑效率. (iii) 一些实验室用 电穿孔方法代替显微注射, 以提高受精卵编辑通量, 避免注射所致损伤, 改善胚胎存活率 ${ }^{[13]}$, 但缺点之 一是Cas9-gRNA复合物用量偏高. 有报道使用nCas9 双切口酶系统代替野生型Cas9制备基因敲人小鼠, 但效率可能不及使用野生型 $\mathrm{Cas} 9^{[14]}$. 目前, 已有多

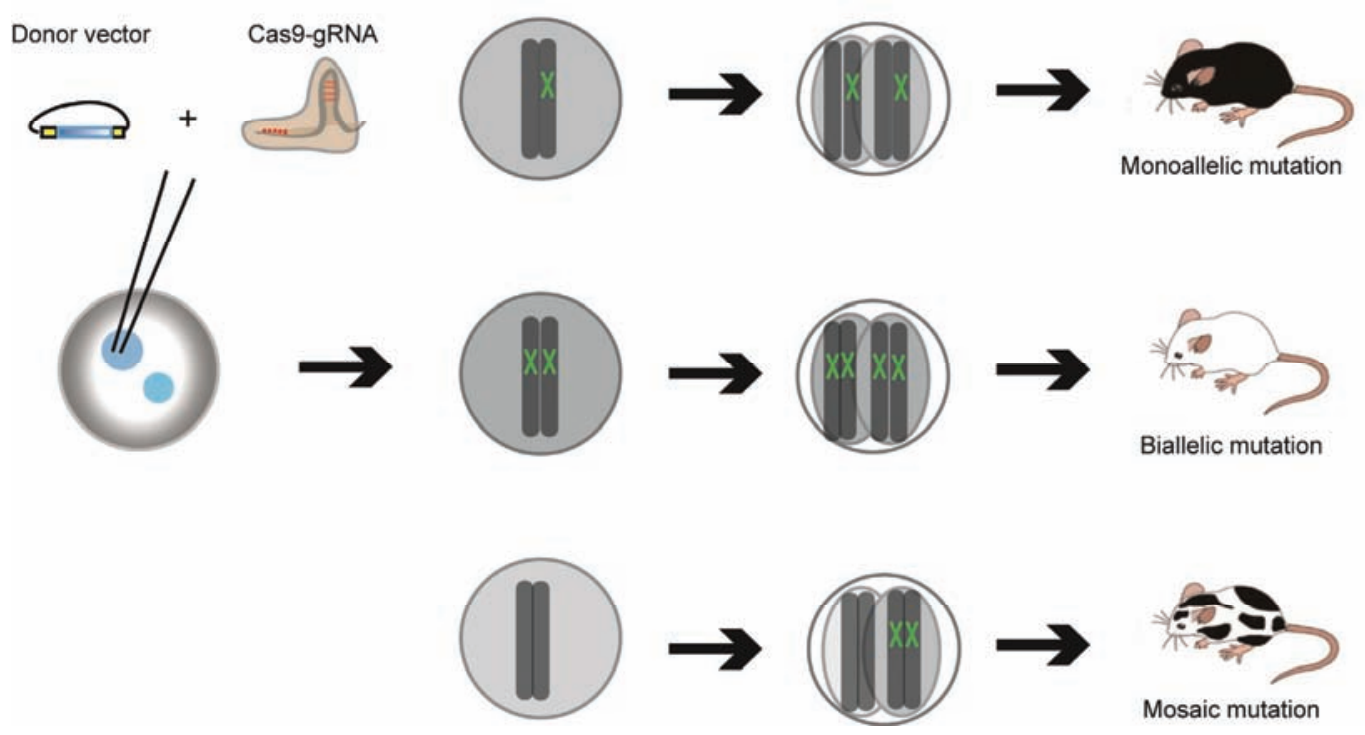

(i)

图 1 受精卵期CRISPR/Cas9 系统介导的基因编辑. 以基因敲人为例, 将Cas9,gRNA, 模板DNA注射至受精卵的雄原核后, 可能会产生 3 种情 况: (i ) 在单细胞期发生单等位基因突变(monoallelic mutation), 得到基因突变杂合子小鼠; (ii) 在单细胞期发生双等位基因突变(biallelic mutation), 得到两个等位基因都发生突变的小鼠; (iii) 在多细胞期发生基因突变, 得到基因突变嵌合体(mosaic mutation)小鼠

Figure 1 Phenotypes and complexity of allele mutations following zygote injection of CRISPR/Cas9 system in mice. CRISPR/Cas9 components (Cas9, gRNA and donor DNA) are injected into the pronuclei of mouse zygotes, which may generate three types of allele modification: ( i ) gene editing occurred in the one-cell stage and mutation appears in one allele (monoallelic mutation); (ii) gene editing occurred in the one-cell stage, but mutations appear in both alleles (biallelic mutation); (iii) gene editing occurred in two or multiple cell stages of embryos, and mutations are produced in a subset of cells (mosaic mutation) 
家生物公司和学术机构常规使用CRISPR/Cas9技术 制备基因修饰小鼠新品系, 将会不断产生新的技术 改进.

\section{2 胚胎期应用}

胚胎期脑部应用CRISPR/Cas9技术的突出优点 是: 此时神经前体细胞数量仍然庞大, HDR活性相对 高, 因而可获得较高的精准编辑效率. 在胚胎期, 一 般采用胎鼠子宫内电穿孔法实施CRISPR/Cas9技术, 通过调整胎鼠脑显微注射部位与电极夹持方向, 将 gRNA-Cas9、模板DNA转送到胎鼠的目标脑区. 在该 方法中, gRNA与Cas9通常整合于单一质粒表达系统, 以降低转染的复杂性. 目前较常用的载体为 $\mathrm{pX} 330$ 质 粒, 该质粒提供了两个 $B b s$ I 酶切位点, 方便导人 $\mathrm{gRNA}$ (图2), 用U6启动子驱动 $\mathrm{gRNA}$ 表达; 在 $\mathrm{gRNA}$
后是广谱的 $\mathrm{CBh}$ 启动子, 驱动人源化 $\mathrm{SpCas} 9$ 核酸酶 表达. 胎小鼠子宫内电穿孔法可在胚胎 10 17日龄 (E10 E17)之间应用. 在成功构建质粒后, 抽提高纯 度的质粒DNA与固绿染料混合, 用玻璃微电极将 1 2 $\mu \mathrm{L}$ DNA混合物注射至胎鼠的单侧脑室，按选定方向 施加方波电脉冲. 电脉冲参数一般为: $35 \sim 45 \mathrm{~V}$, 30 50 ms，4 5次，间隔 $1 \mathrm{~s}$, 在此范围内随肧胎日龄 增加上调参数. 可共转染 $\mathrm{pCAG-EGFP}$ 质粒用于了解 转染效率. Shinmyo 等人 ${ }^{[15]}$ 以E15.5胚胎为例, 详细介 绍了这一技术的实验流程. 子宫内电穿孔实验要避 免误电击胎鼠心脏, 手术通常需在 $1 \mathrm{~h}$ 内完成, 以提 高电转胎鼠的成活率.

\section{3 成年期应用}

在成年期动物, 脑组织的基因导人主要通过注

(a)

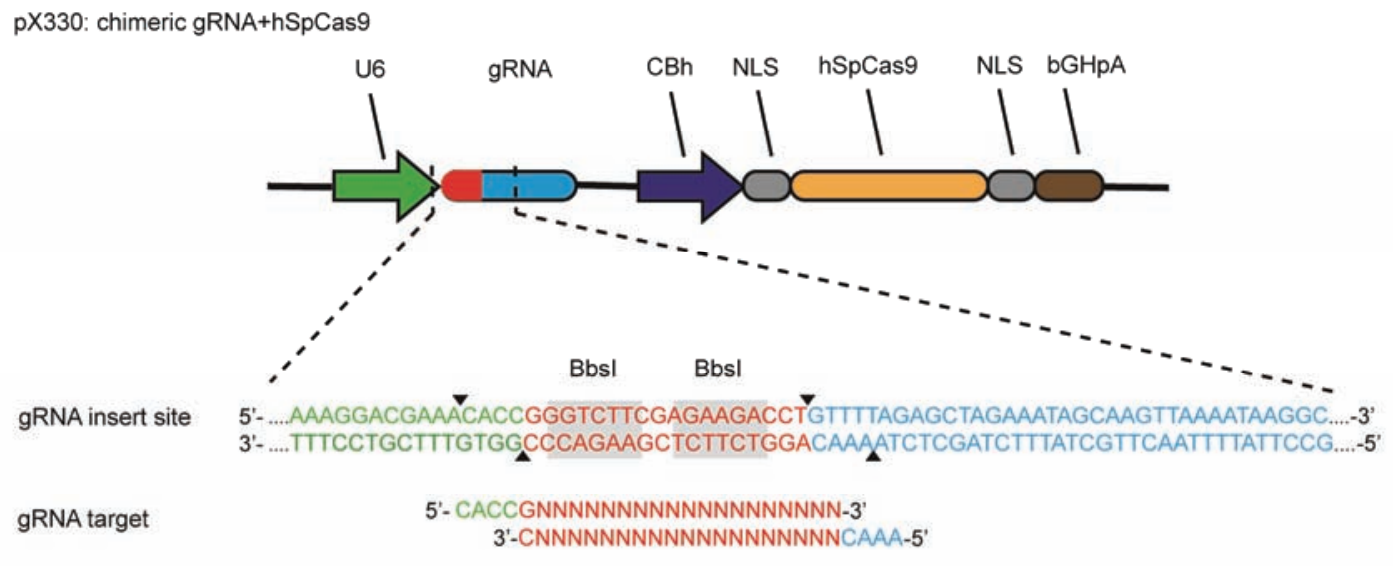

(b)

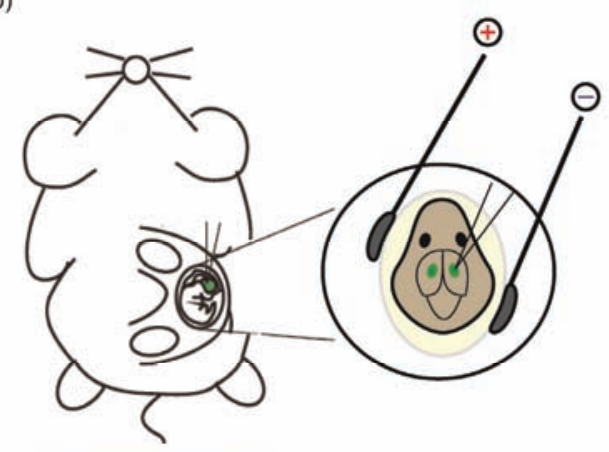

In utero eletroporation

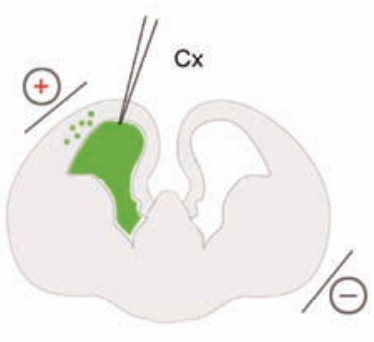

Cortex targeted

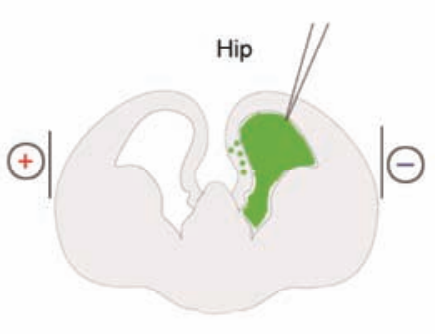

Hippocampus targeted

图 2 胚胎期小鼠应用子宫内电穿孔法在脑部实施CRISPR/Cas9 介导的基因编辑. (a) pX330 质粒表达框示意图, 质粒序列来自Addgene网页. (b) 子宫内电穿孔技术示意图. 利用胚胎期鼠脑的结构特点, 将质粒注射于脑室的不同侧并调整电极位置, 以获得相对的脑区靶向性, 如大脑 皮层 $(\mathrm{Cx})$ 或海马(Hip)

Figure 2 Delivery of CRISPR/Cas9 gene editing system into the mouse brain at the embryonic stage using in utero electroporation. (a) The pX330 plasmid widely used in vivo. The sequence for the construct is adapted from the Addgene web page. (b) Schematics of applying in utero electroporation technique in the brain of mouse embryo. The relative positions of the injection sites and electrode paddles can be adjusted to achieve the targeting of distinct brain regions, such as the cerebral cortex $(\mathrm{Cx})$ or the hippocampus (Hip) 
射病毒的方法来实现. 腺相关病毒 AAV作为一种低 毒性、高效率、可定点整合的载体被广泛应用. Swiech 等人 ${ }^{[16]}$ 详细介绍了如何应用AAV方法在成年小鼠脑 实施 CRISPR/Cas9基因编辑. 以 Mecp 2 基因为例, 将 AAV-SpCas9和AAV-gRNA病毒混合物注射至成年 小鼠海马齿状回，2周后大约 $68 \%$ 的被感染神经元基 因组发生了Mecp2基因编辑, $\mathrm{MeCP} 2$ 总蛋白水平也下 降了 60\%以上. 他们还以Dnmt1, Dnmt $3 a, D n m t 3 b$ 为 例介绍了多基因编辑, 将这3个基因的 gRNA分别插 于U6启动子之后, 再串联起来用 AAV包裹, 注射后 8 周有大约 $35 \%$ 的被感染神经元发生三基因同时编辑. 成年期AAV病毒注射基因编辑方法的有效性随后被 多个研究报告所证实 ${ }^{[17,18]}$. 另一方面, 如果用 LSL (LoxP-Stop-LoxP)元件控制Cas9仅在Cre阳性的神经 细胞中表达, 可以实现条件性基因编辑. 例如, CaMK II $\alpha$-Cre小鼠脑内注射AAV-LSL-Cas9与AAVgRNA病毒, Cas9蛋白仅在CaMK II $\alpha$-Cre阳性神经元 中表达, 因而仅 CaMK II $\alpha$ 启动子活性高的神经元发 生了选择性基因编辑.

AAV 病毒在体应用已展示高效低毒的优点, 但 装载容量相对小, Cas9与 gRNA通常需要分开包装. 有人尝试利用容量较大的整合酶缺陷慢病毒, 试图 将所有基因编辑元件包装进单一载体. 脂质体由于 受细胞毒性和效率方面的限制, 以往多用于体外实 验. 近期, Wang等人 ${ }^{[19]}$ 采用生物可降解的脂质纳米 颗粒包裹负电荷加强的 Cas9-gRNA复合物, 期望构 建改进型脂质体投递系统.

\section{4 神经科学的应用现状}

CRISPR/Cas9技术在脑科学中应用已涉及脑基 因功能分析、基因敲人小鼠构建以及神经系统疾病研 究等多个方面.

\section{1 神经科学基础研究}

研究蛋白脑内分布的传统方法为免疫染色或者 过表达带标签的内源性蛋白. 这些传统方法通常会 面临一些现实问题, 如免疫染色有非特异性交叉反 应, 或者缺少特异性抗体; 过表达则可能改变原蛋白 分布. 即使采用传统的基因敲人小鼠方法, 也常常会 因标记的阳性细胞过密而缺乏亚细胞分辨率. Mikuni 等人 ${ }^{[20]}$ 将内源性蛋白基因与标签蛋白基因融合, 再 通过子宫内电穿孔法将CRISPR/Cas9介导的基因敲
人，建立了一个高分辨率、高反差观察内源性蛋白分 布的方法. 这种技术可以观察定位于细胞核、胞浆、 胞膜、细胞骨架、囊泡的各种蛋白质，可通过调整肧 胎电穿孔的时机与脑区将技术升级至观察不同脑区、 不同类型神经细胞, 可与电子显微镜相结合获得纳 米级的分辨率，还可利用CRISPR/Cas9技术可多基因 编辑的特点同时观察多个蛋白. 总之, 这种基于 CRISPR/Cas9技术的新方法, 可以将内源性蛋白脑内 分布的研究提升至一个前所未有的水准. CRISPR/ Cas9技术如与光遗传学等技术结合, 在神经系统实 现可诱导的、时空特异性的基因编辑, 可应用于脑神 经环路的解析研究.

\section{2 构建基因敲除/敲入小鼠}

用传统技术制备各种基因工程小鼠模型一般费 时较长, 其中某些技术(如Cre/LoxP技术)在实际应用 中十分繁琐. 用CRISPR/Cas9技术实施基因敲除/敲 人绕开了一些常见的限制, 根据不同的研究目的, 大 致分为 3 类基因编辑方案，即基因敲除、基因敲人和 条件性基因敲除(图3).

在受精卵期，用CRISPR/Cas9技术产生基因敲除 小鼠是以NHEJ机制为主. 目前对于这种途径实现基 因敲除的实际效果仍然存有一些不足, 例如敲除效 率低或差异巨大, 突变种类复杂多样, 仅一部分细胞 发生突变等。受精卵单细胞期快速导人有活性的 CRISPR/Cas9系统是一种改进措施, 可减少嵌合体形 成. 在局部组织层面, 用小鼠海马 $\mathrm{AAV}$ 病毒注射 ${ }^{[16]}$ 、 胚胎脑电转染质粒 ${ }^{[21]}$ 实施CRISPR/Cas9 介导的基因 敲除通常可获得较高的敲除效率; 在脑片组织上甚 至实现了完全的双等位基因敲除 ${ }^{[22]}$. 这些研究结果 显示局部脑组织可达到较高的基因敲除效率，推测 是因为 gRNA/Cas 9 在分化后神经元中持久表达, 对 靶基因进行了充分的编辑. 另需指出, 不同实验室报 道的基因敲除效果差异很大，可能与敲除系统、检测 方法等个体因素有关.

应用CRISPR/Cas9系统制备基因敲人小鼠是基 于HDR机制，有方便、快捷的优点，构建时间从常规 方法的1 2年大大缩短至3 6月. 另外, 利用Cas9酶 可以结合不同 gRNA的特性, 可以设计同时敲人多个 基因 ${ }^{[23]}$. CRISPR/Cas9技术也可与其他技术手段结合 起来, 实现可诱导性(如四环素或他莫昔芬)或组织特 异性 (如 C r e 依赖性) 基因敲除/敲人。剑桥大学 
(a) Knock-out

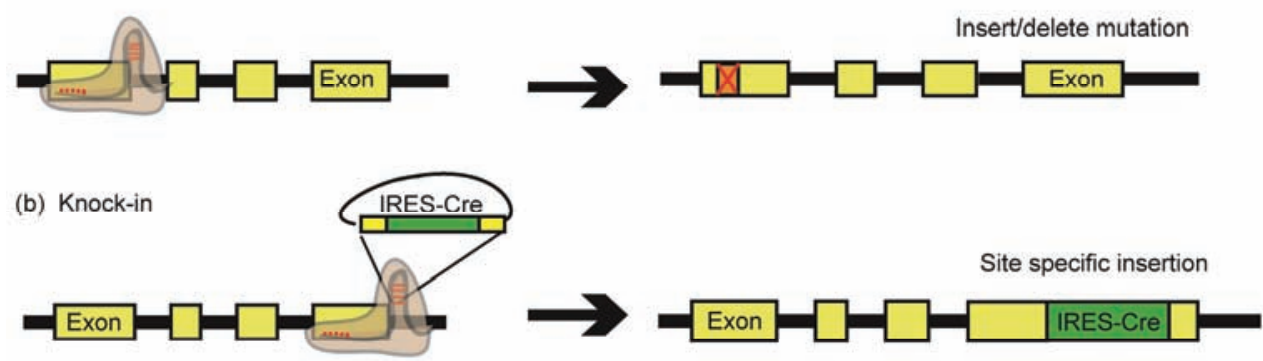

(c) Conditional and reversible knock-out

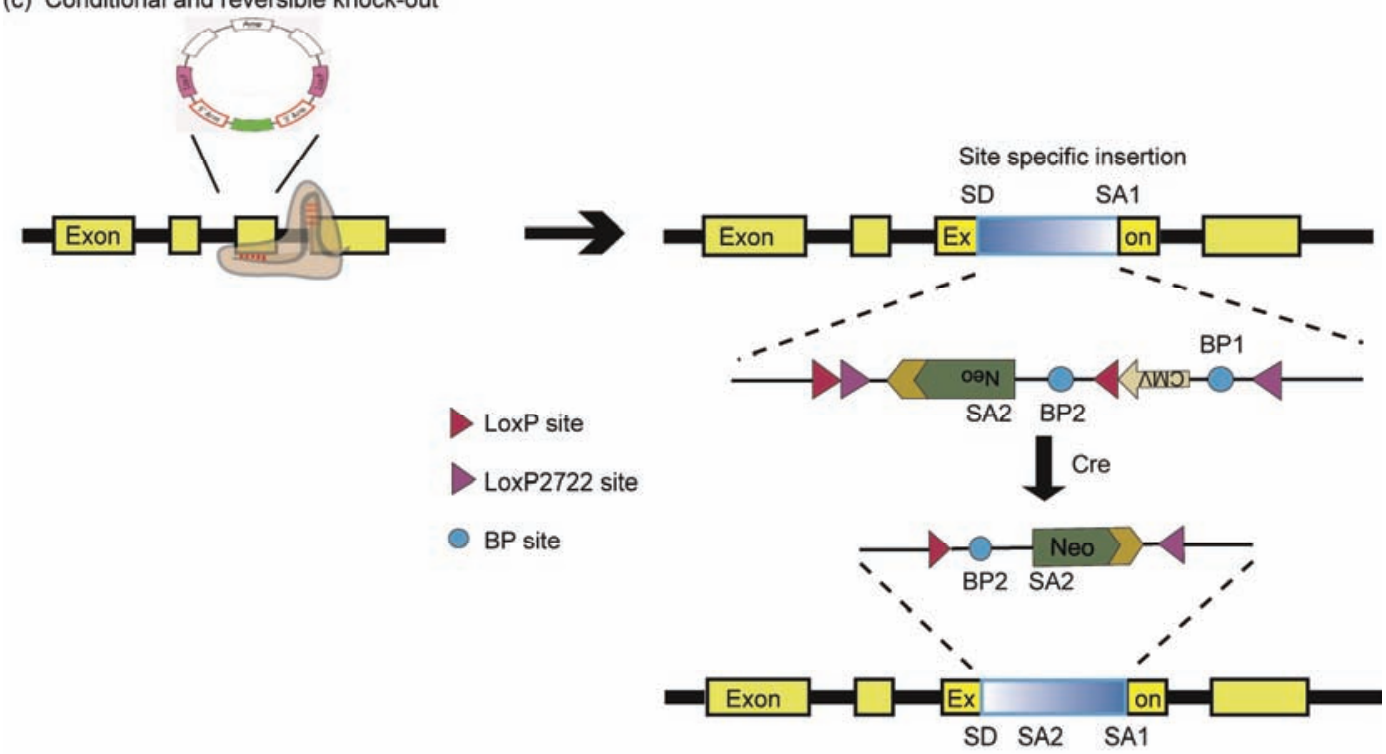

Cre-dependent Knock out

图 3 CRISPR/Cas9 技术用于制备基因敲除/敲人小鼠的 3 种常见方案. (a) 基因敲除. 该方案通过将Cas9 蛋白, gRNA二者同时导人受精卵, Cas9 在 gRNA识别的基因靶点切割DNA双链, 细胞主要通过非同源性末端接合机制修复双链断裂, 但易产生插人、缺失、倒位等复杂突变, 干 扰靶基因的正常表达. (b) 基因敲人. 该方案在系统中引人了含同源臂的供体模板, 细胞主要通过同源重组机制修复DNA双链断裂, 可将目的 基因(以Cre为例)精确地插人到设计位点. (c) 条件性基因敲除: 该方案利用基因表达调控中的内含子剪切机制, 将篮选基因反向插人到目的基 因中, 由于采用内含子方式, 不影响目的基因表达. 内含子中有双LoxP位点包裹的抗性或者苂光报告基因表达框, 在引入Cre重组酶(注射或 Cre小鼠)后, LoxP位点之间的篮选基因表达框翻转，破坏了目的基因的表达，实现条件性敲除. 该系统可以升级为条件性基因敲人. IRES，内 部核糖体进入位点(internal ribosome entry site); SD, 剪接供体(splicing donor); SA1 和SA2, 剪接受体(splicing acceptor); BP1 和BP2, 分支点 (branching point)

Figure 3 General approaches by using the CRISPR/Cas9 system to generate genetically modified mice. (a) Knock-out: by CRISPR/Cas9 microinjection into the mouse zygotes, the cells repair the DNA double-strand breaks mainly through non-homologous end joining mechanism which probably cause frame-shift insertion and deletion (indel) mutations. (b) Knock-in: this approach introduces a donor template with two homologous arms. In this case, the double-strand breaks are mainly repaired by homology directed repair mechanism and the template sequence is precisely inserted into the targeted site. (c) Inducible and conditional knock-out: this approach uses the gene expression regulation of the intron cleavage mechanism. The reporter gene is inserted into the target gene as an intron, therefore, this insertion does not affect the target gene expression. When introducing Cre recombinase (by injection or breeding with Cre mice), the double LoxP sites will reverse the screening gene and simultaneously destroy the expression of the target gene, to achieve conditional knock-out. IRES, internal ribosome entry site; SD, splicing donor; SA1 and SA2, splicing acceptor; BP1 and BP2, branching point

Andersson-Rolf等人 ${ }^{[24]}$ 将CRISPR/Cas9技术与可翻转 的打靶载体FLIP相结合, 通过共同导人CRISPR/Cas9 系统与普适的条件性内含子盒, 建立了CRISPRFLIP一步法，用于细胞层面可诱导性基因敲除实验，
并可升级至制备条件性基因敲除/敲人小鼠.

\section{3 神经系统疾病研究}

由于CRISPR/Cas9技术有高效、普适的特点, 已 
被尝试用于编辑某些遗传性或病毒性疾病的致病基 因. 脆性 $\mathrm{X}$ 综合征是由于 $\mathrm{X}$ 染色体上 $F M R I$ 基因突变 所致的遗传性疾病, 表现为智力低下等症状. FMRI 基因的典型突变为 $5^{\prime}-\mathrm{UTR}$ 区的 $\mathrm{CGG}$ 序列重复次数过 多(如200次以上), 最终使 $F M R 1$ 基因启动子上游序列 异常甲基化, FMRI基因失活. Park等人 ${ }^{[25]}$ 用 $C G G$ 重 复区上游 -17 - 15 位 GGG 序列作为 PAM 设计了 gRNA, 并将此gRNA/Cas9质粒转染至脆性X综合征 患者来源的iPS细胞, 虽然只获得 2\% 3\% 的编辑效 率, 但阳性克隆完全删除了 FMRI基因的 $\mathrm{CGG}$ 重复序 列, 伴随该基因启动子上游 $\mathrm{CpG}$ 岛显著的脱甲基化, $F M R 1$ 基因转录功能恢复. 跟踪研究还发现, 被 Cas9 编辑校正的iPS细胞在诱导为成熟神经元之后, FMRI 基因依然持续表达. 这项实验提示了CRISPR/Cas9技 术在治疗某些单基因遗传性疾病中有较大潜力. 与 之类似, 亨廷顿病(HD)的致病相关基因 $H T T$ 位于 4 号 常染色体的短臂, 其编码区 CAG串联重复序列异常 扩展至 40 个以上将导致疾病. 有细胞水平的研究报 道, 在 CAG 重复区内由 ZFN 或 CRISPR/Cas9产生 DNA双链断裂都引起CAG重复数量继发增减双向变 化; nCas9切口酶主要减缩 $\mathrm{CAG}$ 重复数量, 但此效应 依赖于DNA损伤反应激酶, 而与单链断裂修补机制 无关 ${ }^{[26]}$.

CRISPR/Cas9技术已用于在体水平的治疗尝试. 美国加利福尼亚州Cedars-Sinai医学中心王少梅实验 室 ${ }^{[27]}$ 在视网膜色素变性模型S334ter-3大鼠上试验了 CRISPR/Cas9介导的在体基因编辑疗效. S334ter转基 因大鼠携带了视紫红质基因的一种显性突变S334ter $\left(R h o^{\mathrm{S} 334}\right)$, 包括334位丝氨酸密码被一个终止密码子 替代和羧基端 15 个氨基酸密码的缺失, 模型鼠表现 为出生后视网膜外核层感光受体进行性丧失, 视觉 功能严重下降. 在模型大鼠出生当天, 一次性向单侧 视网膜下区注射靶向 $R h o^{\mathrm{S} 334}$ 的 gRNA-Cas9质粒加电 转染, 便可成功失活 $R h o^{\mathrm{S} 334}$ 突变基因, 阻遏后期的视 网膜退化, 改善视觉灵敏度. 美国Emory大学李晓江 实验室 ${ }^{[28]}$ 在亨廷顿病小鼠模型上试验了CRISPR/ Cas9基因编辑对该病的疗效. HD140Q基因敲人小鼠 在对应的第一个外显子中插人了含 140 个 $\mathrm{CAG}$ 重复 的人 HTT 基因, 纹状体神经元核内 $\mathrm{mHTT}$ 突变蛋白在 4 6月龄时开始上升，9 10月龄时形成明显的聚集. 此研究设计了两个串联的 gRNA分别靶向 $C A G$ 重复 的两端侧翼区, 与 $\mathrm{Cas} 9$ 分别包装在两个 $\mathrm{AAV}$ 病毒载
体中, 病毒混合物注射到 3 或 9 月龄的纯合子模型小 鼠单侧纹状体. 结果显示, 用CRISPR/Cas9方法治疗 可以持久抑制纹状体神经元 $m H T T$ 突变蛋白表达, 排 空突变蛋白聚集, 减轻早期神经病理表现, 改善模型 小鼠的运动功能. 实际上, 蛋白异常聚集是多种神经 退行性疾病的一类共同病理特征，例如在帕金森病 和Lewy痴呆患者脑中 $\alpha$-synuclein蛋白聚集，阿尔茨 海默病和额颞叶痴呆患者脑中 tau蛋白聚集. 采用 CRISPR/Cas9技术去除此类异常蛋白的表达，能否延 缓相关神经退行性疾病的进展, 将是未来的研究焦 点之一.

\section{CRISPR/Cas9技术的发展方向}

虽然CRISPR/Cas9技术在编辑基因组上比传统 技术更为简便、高效，但在多个方面仍然存在不足或 发展潜力, 需要继续改进与提升.

\section{1 提高非分裂细胞的精确编辑效率}

同源重组HDR主要发生在分裂细胞, 即使添加 了同源臂，重组效率依然不高. 在分化后的非分裂细 胞如成熟神经元，如何提高CRISPR/Cas9系统对基因 组的精确编辑效率是十分重要的现实问题. 近期, Salk研究所Belmonte实验室 ${ }^{[29]}$ 改进了之前以NHEJ为 基础的编辑方法, 建立了一个称为同源非依赖性靶 向嵌人 (homology-independent targeted integration, HITI)方法. HITI方法主要做了如下优化：供体载体 中去除细菌性骨架和polyA序列减少敲人基因沉默, 供体载体缩小甚至成微环状又提高了目的基因插人 效率; 供体DNA两端正向包绕 gRNA/Cas9靶序列作 为切割位点, 保证供体DNA以正确方向插人基因组. Cas9融合了核定位信号BPNLS或SV40NLS二分体. HITI方法的编辑效率比同源重组方法高, 又保持了 编辑的精确性, 适用于非分裂细胞的基因编辑. 将 HITI-AAVs混合物注射至10周龄小鼠视皮层，2周后 相对编辑效率(占感染细胞数)为 $10.6 \%$, 绝对编辑效 率(占细胞总数)为 $3.5 \%$. 该研究小组还在视网膜色素 变性的自发模型RCS(皇家外科学院)大鼠中测试了这 一技术的有效性. RCS大鼠的视网膜变性是由Mertk 基因 $1.9 \mathrm{~kb}$ 纯合子缺失突变所致. 将含Mertk基因缺 失片段的HITI-AAVs混合物注射到3周龄模型大鼠的 视网膜下区, 7 8周龄时观察到Mertk基因表达水平和 视网膜外核层厚度均部分恢复，视网膜电图光反应 
也有小幅改善. 中国科学院神经科学研究所杨辉实 验室则从优化HDR方向改进, 在 $800 \mathrm{bp}$ 同源臂的两端 添加 gRNA/Cas9切割位点, 这一方法改变虽然在小 鼠胚胎干细胞上对基因编辑效率影响不大, 但在星 型胶质细胞和原代神经元上基因编辑效率有显著提 高 ${ }^{[30]}$. 作者已将这个改进方法应用于猴胚胎和成年 小鼠脑的基因编辑. 微同源介导的末端接合 (microhomology-mediated end joining, MMEJ)采用5 25 bp的 短同源臂, 但在培养细胞上显示低编辑效率 ${ }^{[31]}$. 最 近, Quadros等 ${ }^{[32]}$ 将同源臂改为90nt左右的单链, 在小 鼠受精卵期导人展示了较高的编辑效率. 这方面的不 断改进将可能产生更加高效、精确的基因编辑方法.

\section{2 原位修复单碱基突变}

一些遗传性疾病是由碱基点突变引起, 但以往 的点突变编辑方法效率较低. 近年来又报道了几种 新的“碱基编辑器(base editor)”, 其基本思路是将胞 嘧啶脱氨酶与DNA识别蛋白融合. 例如, 选用nCas9 切口酶或无活性的 dCas9作为DNA识别蛋白, 两者仍 通过gRNA识别和结合基因靶点, 但不产生DNA双链 切割, 而是由胞嘧啶脱氨酶催化胞嘧啶转化为尿嘧 啶 $^{[33]}$. Kim等人 ${ }^{[34]}$ 在全基因组范围检查了一种碱基编 辑器 (rAPOBEC1-nCas9-UGI, 脱氨酶-Cas9切口酶尿嘧啶DNA糖基化酶抑制蛋白)的靶点特异性, 发现 单个 gRNA在人类全基因组仅产生少量的碱基转换位 点, 提示碱基编辑特异性良好. 另外还发现, nCas9编 辑器的碱基编辑效率高于 $\mathrm{dCas}$ 编辑器, 但同一 $\mathrm{gRNA}$ 诱导的碱基编辑活性与它诱导的Cas9核酸酶活性之间 并不平行, 两个系统的 gRNA错配耐受也不相同.

\section{3 删除DNA片段的长度限制}

基因的大片段缺失突变也被鉴定为某些人类疾 病的可能病因. 为了模拟这种大片段缺失的情况, 需 要构建长度达几个 $\mathrm{Mb}$ (百万碱基对)的缺失突变小鼠 作为研究模型. 以往, 用受精卵显微注射CRISPR/ Cas9系统常规方法所获得的基因缺失突变小鼠, 报 道最长的缺失片段为 $1.2 \mathrm{Mb}$. 日本学者 Kato等人 ${ }^{[35]}$ 试验了将两条gRNA(靶向基因大片段的两端)与Cas9 蛋白共同注射至小鼠受精卵原核. 这种方法可以产 生长达 $5 \mathrm{Mb}$ 的缺失突变胚胎, 但突变效率不高或不 稳定, 阳性胚胎的双等位基因缺失突变仅出现于一 部分细胞.

\section{4 “脱靶”效应受关注}

脱靶效应是由于 $\mathrm{gRNA}$ 与 DNA 靶序列之间允许 有个别碱基错配. 目前, gRNA序列的脱靶概率可以 通过生物信息学手段加以预测. 例如, 麻省理工学院 张峰实验室的网站可帮助用户设计目标基因的 gRNA, 在给出推荐 gRNA序列的同时, 也预测了脱 靶的可能序列. 通过优化 $\mathrm{gRNA}$ 的设计与应用条件, 包括开发高保真的核酸酶, 可以显著提高 CRISPR/ Cas9系统的特异性. CRISPR/Cas9技术存在潜在“脱 靶”效应的程度一直有争议, 但近期的多项研究显示, 在体应用CRISPR/Cas9基因编辑技术诱发脱靶效应 的概率并不高 ${ }^{[36,37]}$. 然而, CRISPR/Cas9技术已开始 用于人体临床试验, 安全问题需极其慎重. CRISPR/ Cas9系统的“脱靶”程度以及对于基因组稳定性的潜 在影响, 未来仍需通过大样本、多中心、严谨深人的 基础和临床研究来澄清.

\section{5 定向导人神经系统的问题}

成年动物在体导人 CRISPR/Cas9系统有病毒方 式与非病毒方式, 可采取局部注射或全身注射 ${ }^{[38]}$. 脑科学应用CRISPR/Cas9技术首先需要实现脑靶向, 然后再进一步靶向特定的神经细胞类型或细胞群. 美国加州理工学院的 Deverman等人 ${ }^{[39]}$ 用一种基于 Cre重组的AAV病毒衣壳定向进化方法, 笁选出一个 AAV变种AAV-PHP.B, 具有更好的脑靶向特性, 成 年小鼠静脉注射后向脑内传送目的基因的能力比以 往的AAV9提高40倍以上. 结合已有的可诱导性基因 操作系统, 选择性调控细胞内Cas9核酸酶表达, 有可 能实现神经细胞类型特异性基因编辑, 但这方面的 研究目前还很少. 实际上, 已公布的CRISPR/Cas9临 床治疗实验基本上是采用活体细胞体外编辑后再回 输的方式 (exvivo). 未来, 如何实现高效、安全的 CRISPR/Cas9体内递送(in vivo), 仍然是一个具有挑 战性的难题. 目前研发中的 AAV 递送系统, 尚待严 谨的临床试验来鉴定成效.

\section{6 结束语}

目前的CRISPR/Cas9基因编辑技术较报道初期 在多个方面已有了显著的改进和扩展, 在神经科学 领域也得到了一些初步应用. 未来, 将CRISPR/Cas9 技术与光遗传学、药物遗传学、多光子成像、在体神 
经活动记录等先进技术相结合，会进一步提升神经 科学工作者研究复杂脑环路与脑疾病的能力. 随着
逐步建立高效可控的脑靶向输送系统，CRISPR/Cas9 技术尚有应用于某些神经系统疾病治疗的良好潜力.

\section{参考文献}

1 Capecchi M R. Altering the genome by homologous recombination. Science, 1989, 244: 1288-1292

2 Hsu P D, Lander E S, Zhang F. Development and applications of CRISPR-Cas9 for genome engineering. Cell, 2014, 157: 1262-1278

3 Burstein D, Harrington L B, Strutt S C, et al. New CRISPR-Cas systems from uncultivated microbes. Nature, 2017, 542: 237-241

4 Gao L, Cox D B T, Yan W X, et al. Engineered Cpf1 variants with altered PAM specificities. Nat Biotechnol, 2017, 35: 789-792

5 Xu S, Cao S, Zou B, et al. An alternative novel tool for DNA editing without target sequence limitation: The structure-guided nuclease. Genome Biol, 2016, 17: 186

6 Ishino Y, Shinagawa H, Makino K, et al. Nucleotide sequence of the iap gene, responsible for alkaline phosphatase isozyme conversion in Escherichia coli, and identification of the gene product. J Bacteriol, 1987, 169: 5429-5433

7 Jinek M, Chylinski K, Fonfara I, et al. A programmable dual-RNA-guided DNA endonuclease in adaptive bacterial immunity. Science, 2012, 337: 816-821

8 Salsman J, Dellaire G. Precision genome editing in the CRISPR era. Biochem Cell Biol, 2017, 95: 187-201

9 Kleinstiver B P, Prew M S, Tsai S Q, et al. Engineered CRISPR-Cas9 nucleases with altered PAM specificities. Nature, 2015, 523: $481-485$

10 Jinek M, Jiang F, Taylor D W, et al. Structures of Cas9 endonucleases reveal RNA-mediated conformational activation. Science, 2014, 343: 1247997

11 Sternberg S H, LaFrance B, Kaplan M, et al. Conformational control of DNA target cleavage by CRISPR-Cas9. Nature, 2015, 527: $110-113$

12 Maruyama T, Dougan S K, Truttmann M C, et al. Increasing the efficiency of precise genome editing with CRISPR-Cas9 by inhibition of nonhomologous end joining. Nat Biotechnol, 2015, 33: 538-542

13 Chen S, Lee B, Lee A Y, et al. Highly efficient mouse genome editing by CRISPR ribonucleoprotein electroporation of zygotes. J Biol Chem, 2016, 291: 14457-14467

14 Raveux A, Vandormael-Pournin S, Cohen-Tannoudji M. Optimization of the production of knock-in alleles by CRISPR/Cas9 microinjection into the mouse zygote. Sci Rep, 2017, 7: 42661

15 Shinmyo Y, Kawasaki H. CRISPR/Cas9-mediated gene knockout in the mouse brain using in utero electroporation. Curr Protoc Neurosci, 2017, 79: 3.32.1-3.32.11

16 Swiech L, Heidenreich M, Banerjee A, et al. In vivo interrogation of gene function in the mammalian brain using CRISPR-Cas9. Nat Biotechnol, 2015, 33: 102-106

17 de Solis C A, Ho A, Holehonnur R, et al. The development of a viral mediated CRISPR/Cas9 system with doxycycline dependent gRNA expression for inducible in vitro and in vivo genome editing. Front Mol Neurosci, 2016, 9: 70

18 Murlidharan G, Sakamoto K, Rao L, et al. CNS-restricted transduction and CRISPR/Cas9-mediated gene deletion with an engineered AAV vector. Mol Ther Nucleic Acids, 2016, 5: e338

19 Wang M, Zuris J A, Meng F, et al. Efficient delivery of genome-editing proteins using bioreducible lipid nanoparticles. Proc Natl Acad Sci USA, 2016, 113: 2868-2873

20 Mikuni T, Nishiyama J, Sun Y, et al. High-throughput, high-resolution mapping of protein localization in mammalian brain by in vivo genome editing. Cell, 2016, 165: 1803-1817

21 Straub C, Granger A J, Saulnier J L, et al. CRISPR/Cas9-mediated gene knock-down in post-mitotic neurons. PLoS One, 2014, 9: e105584

22 Incontro S, Asensio C S, Edwards R H, et al. Efficient, complete deletion of synaptic proteins using CRISPR. Neuron, 2014, 83: $1051-1057$

23 Yang H, Wang H, Jaenisch R. Generating genetically modified mice using CRISPR/Cas-mediated genome engineering. Nat Protoc, 2014, 9: $1956-1968$

24 Andersson-Rolf A, Mustata R C, Merenda A, et al. One-step generation of conditional and reversible gene knockouts. Nat Methods, 2017, 14: 287-289

25 Park C Y, Halevy T, Lee D R, et al. Reversion of FMR1 methylation and silencing by editing the triplet repeats in fragile X iPSC-derived neurons. Cell Rep, 2015, 13: 234-241 
34 Kim D, Lim K, Kim S T, et al. Genome-wide target specificities of CRISPR RNA-guided programmable deaminases. Nat Biotechnol, 2017, 35: 475-480

35 Kato T, Hara S, Goto Y, et al. Creation of mutant mice with megabase-sized deletions containing custom-designed breakpoints by means of the CRISPR/Cas9 system. Sci Rep, 2017, 7: 59

36 Hay E A, Khalaf A R, Marini P, et al. An analysis of possible off target effects following CAS9/CRISPR targeted deletions of neuropeptide gene enhancers from the mouse genome. Neuropeptides, 2017, 64: 101-107

37 Midic U, Hung P H, Vincent K A, et al. Quantitative assessment of timing, efficiency, specificity, and genetic mosaicism of CRISPR/Cas9 mediated gene editing of hemoglobin beta gene in rhesus monkey embryos. Hum Mol Genet, 2017, 26: 2678-2689

38 Mout R, Ray M, Lee Y W, et al. In vivo delivery of CRISPR/Cas9 for therapeutic gene editing: progress and challenges. Bioconjug Chem, 2017, 28: 880-884

39 Deverman B E, Pravdo P L, Simpson B P, et al. Cre-dependent selection yields AAV variants for widespread gene transfer to the adult brain. Nat Biotechnol, 2016, 34: 204-209 


\title{
Strategy for applying CRISPR/Cas9 gene editing technology in neuroscience
}

\author{
ZHANG QiPeng ${ }^{1,2}$ \& SUN AnYang ${ }^{1 *}$ \\ ${ }^{1}$ Laboratory of Neurodegenerative Diseases \& Molecular Imaging, Shanghai University of Medicine \& Health Sciences, Shanghai 201318, China; \\ ${ }^{2}$ School of Life Sciences, Nanjing University, Nanjing 210023, China \\ * Corresponding author, E-mail: sunay@sumhs.edu.cn
}

The gene editing technology enables cutting or/and inserting the target gene precisely. The CRISPR (clustered regularly interspaced short palindromic repeats)/Cas9 (CRISPR-associated protein 9) system relies on two major elements, a guide RNA (gRNA) that recognizes a specific DNA sequence, and a nuclease Cas9 that cuts the target DNA and creates double-strand breaks (DSBs). Among a few gene-editing technologies available, CRISPR/Cas9 system has showed tremendous advantages over the others, mainly due to high efficiency of Cas 9 and simple design of gRNA. As a versatile and powerful tool for genome engineering, the CRISPR/Cas9 technology has been used to generate genetically modified mice with unprecedented simplicity and speed, simply by timed delivery of Cas9/gRNA ribonucleoproteins (RNPs) into pronuclear-stage zygotes. The modified protocol introduces Cas9 protein instead of Cas 9 mRNA by rapid electroporation, which enables the gene editing occurring before the first replication of the mouse genome, thus generating non-mosaic mutant embryos. Furthermore, it could be used to produce multiple gene mutations in a single mouse by co-delivering several gRNAs targeted to different genes. Herein, we summarize current applications of CRISPR/Cas9 technology in the field of neuroscience, and aim to provide concise information and perspectives for better utilizing this technology. In brain research, the CRISPR/Cas9 system can be applied either at the one-cell stage of the fertilized eggs in the form of Cas9/gRNA ribonucleoproteins, or at the stage of embryos or adults in the form of plasmid DNA or the viral vectors (commonly AAV variants) respectively. To deliver the system specifically into the developmental or adult brain, in utero electroporation, or stereotaxic injection is commonly employed. Several reports show that AAV-assisted Cas9/gRNA system could achieve a satisfied efficiency for genome editing in the adult mouse brain, but results vary depending on Cas9 activity, gRNA design, detection methods, and the condition of endogenous DNA repair mechanisms. Remarkable efforts have also been made to enhance the incidence of homology-directed repair for precise gene modifications. Regarding therapeutic genome editing, a couple of recent in vivo studies demonstrate that CRISPR/Cas9 system could contract or remove disease-causing alleles in animal models of certain hereditary diseases such as retinitis pigmentosa and Huntington's disease, raising hope for translating therapeutic genome editing to clinical patients. In addition, we discuss major challenges and critical improvements for this technology, including a few modifications for promoting precise editing in non-dividing cells in the case of adult brain. In the future, application of CRISPR/Cas9 technology would be enhanced greatly in neuroscience by developing cell type-specific, timed delivery system in vivo and by combining with other powerful techniques for dissecting brain neural circuits in health and disease.

\section{CRISPR/Cas9, gene editing, knock-out, knock-in, neuroscience}

doi: 10.1360/N972017-00670 